\title{
Importance of Classification Algorithms in Epileptic Seizure Detection
}

Sivakumaran $\mathrm{N}^{1^{*}}$, Sathish $\mathrm{E}^{1}$, Radhakrishnan $\mathrm{K}^{2}$, Raghavan $\mathrm{S}^{3}$, Rajajeyakumar $\mathrm{M}^{4}$, Radhakrishnan $\mathrm{TK}^{5}$, Hemalatha Karnan ${ }^{1}$ and Sankaranarayanan $\mathrm{S}^{1}$

${ }^{1}$ Department of Instrumentation and Control Engineering, National Institute of Technology, Tiruchirappalli, India

${ }^{2}$ Department of Neurology, Amrita Institute of Medical Sciences, Kochi, Kerala, India

${ }^{3}$ Department of Electronics and Communication Engineering, National Institute of Technology, Tiruchirappalli, India

${ }^{4}$ Department of Physiology, Chennai Medical College Hospital and Research Centre, (SRM Group), Irungalur, Trichy, Tamilnadu, India

${ }^{5}$ Department of Chemical Engineering, National Institute of Technology, Tiruchirappalli, India

"Corresponding author: Sivakumaran N, Associate Professor and Head of the Department, Dept. of Instrumentation and Control Engineering (ICE), National Institute of Technology, Trichy, India, Tel : 9443745705; Email ID: nsk@nitt.edu

Rec date: June 3, 2016; Acc date: June 5, 2016; Pub date: June 15, 2016

Copyright: (c) 2016 Sivakumaran N, et al. This is an open-access article distributed under the terms of the Creative Commons Attribution License, which permits unrestricted use, distribution, and reproduction in any medium, provided the original author and source are credited.

\section{Introduction}

Epilepsy is a chronic condition where the brain generates erratic signals leading to tremors [1]. In layman terms epilepsy is known as fits. Statistical studies poses an impact, that more than 70 million of human population are prone to epileptic seizures [2]. Automated epileptic seizure analysis refers to the methods for seizure detection, seizure prediction, and seizure source localization. The challenging area is locating the ictal spikes and seizures during Electro encephalogram (EEG) recording. Features defining the characteristics of the clinical ailment epilepsy is the thriving sector of research in neurophysiology. The classification and characterization algorithms for detecting seizure episodes in patients are vital in this field. The work undertaken renders a hand to the patients and radiologists in detecting the occurrence of seizure. In the current scenario clinicians analyse video EEG for a prolonged duration (even a week) to diagnose the seizure events. At the moment of such a paradigm an alarm circuit is used to notify the clinicians for a prompt medical aid to the victim. Therefore an approach is handled to computationally automate seizure marking and alert the doctors at the time of seizures. The types of feature identification utilizes two different automated methods for analysis of EEG recordings: inter-ictal spike detection and epileptic seizure analysis and characterization.

\section{Filtering Techniques}

The epileptic seizures are identified by eliminating the challenges in the signal [3]. The emphasis is given on filtering the EEG signals from artefacts using signal processing and filtering techniques like band pass filtering, notch filtering, wavelet filtering and independent component analysis. The mode of filtering is based upon the subject's age group, sensitivity of the EEG recorder, the signal recording ambience, the type of epilepsy and also due to various cortical sectors of the brain.

\section{Feature Extraction}

The next motive is to identify the parameters within the filtered EEG signal which notifies the physiological impact of the epileptic seizures. These parameters are known to be the essential features which are to be extracted from the filtered EEG signal for the accurate identification of epileptic seizures. There are different methodologies in hand for statistical feature extraction viz. statistical features, discrete Fourier Transforms, Wavelet Transforms and Hilbert transforms [4].

\section{Feature selection}

Even though it is an optional layer in classification process, feature selection is a widely acclaimed technique among the researchers in order to shrink the dimensionality of the large dataset and ultimately to improve the rate of detection and classification. Least Angle Regression, Leave-One-Out, wrapped Sparse Group Lasso, minimum Redundancy Maximum Relevance, t-test, conventional and successful global optimization algorithm are used in various literature works for selecting optimal features for the classification process $[5,6]$.

\section{Seizure Detection}

Automated techniques are implemented to detect the seizure occurrence instances from the recorded signal [7]. The advantage of the automated detection, is the data handling and manipulation of large dimension signals with multiple seizure occurrence points. The ultimate motto of the seizure detection technique is to conceal the information underlying in the signal to raise an indication about the occurrence of the seizure with utmost accuracy.

Different seizure detection criterion can be divided into nine categories as follows.

- Mimetic techniques, which is basically an traditional recognition technique.

- Morphological analysis, which in incorporates slow waves, spikes and poly spikes decomposition.

- Template matching algorithms, also known an pattern recognition.

- Parametric approaches.

- Independent component analysis.

- Artificial neural networks.

- Clustering techniques.

- Data mining and other classification techniques.

- Knowledge-based rules.

On the other hand video EEG recordings does not show the impact of seizures due to muscular movements, eye-blinks, muscular twitches and also seizure during sleep. The flow of the automated EEG signal based seizure detection is given by Figure 1 . 
Citation: Sivakumaran N, Sathish E, Radhakrishnan K, Raghavan S, Rajajeyakumar M, et al. (2016) Importance of Classification Algorithms in Epileptic Seizure Detection. J Bioeng Biomed Sci 6: 193. doi:10.4172/2155-9538.1000193

Page 2 of 2

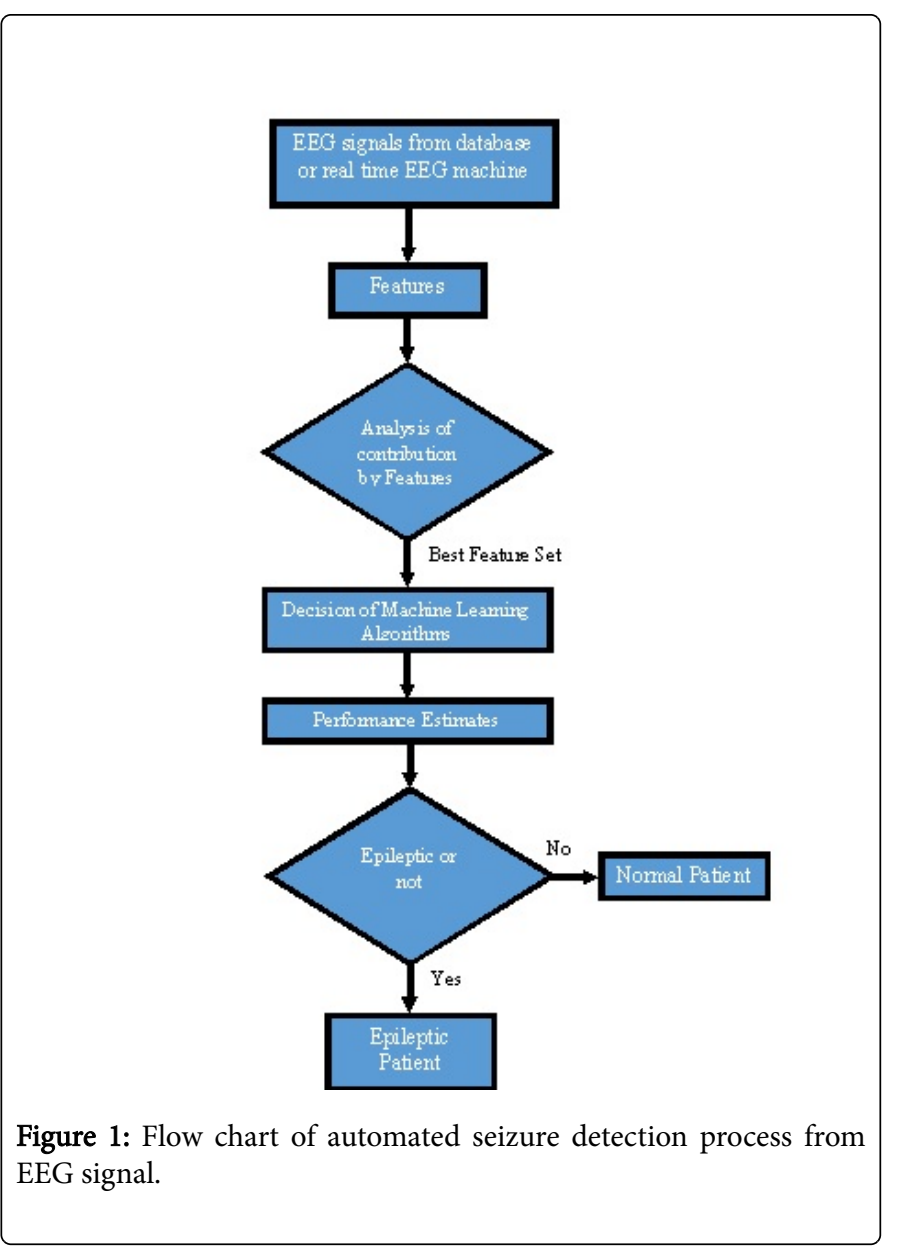

\section{Conclusion}

Locating epileptic seizures manually over a large dimension data of the recorded EEG signal is a cumbersome task and demands lots of time and computational space for analysing a comparatively a small window of the signal. This may also lead to the manual misconceptions and overseeing at some seizure occurrence instances. These difficulties have motivated the development of automated methods that identifies the seizure events, and present to a neurophysiologist epochs containing epileptic events. The various techniques involved in literature for filtering, feature extraction, feature selection and classifier design algorithms were discussed in brief way. Selection of these techniques depends on various parameters like applicability, nature of research problem, ease in implementation and time consumption. The main goal behind this article is to assist the researchers in the field of EEG signal analysis to understand the available methods and also adopt the same for the detection of neurological disorders associated with EEG recordings.

\section{References}

1. Moshé SL, Perucca E, Ryvlin P, Tomson T (2014) Epilepsy: new advances. Lancet.

2. Fazel S, Wolf A, Långström N, Newton CR, Lichtenstein P (2013) Premature mortality in epilepsy and the role of psychiatric comorbidity: a total-population study. Lancet 382: 1646-1654.

3. Lotte F (2014) A tutorial on EEG signal-processing techniques for mental-state recognition in brain-computer interfaces. Guide to BrainComputer Music Interfacing 133-161.

4. Najarian K, Splinter R (2012) Biomedical Signal and Image Processing, (2nd edn). CRC press, Florida USA.

5. Sen B, Peker M, Celebi F V and Cavusoglu A (2014) A comparative study on classification of sleep stage based on EEG signals using feature selection and classification algorithms. J Med Syst 38(3):18

6. Bermúdez RG, Laencina GPJ, González RJ, Dorda RJ (2013) Efficient feature selection and linear discrimination of EEG signals. Neurocomputing 115:161-165

7. Ramgopal S, Thome-Souza S, Jackson M, Kadish N E, Fernndez I S et al. (2014) Seizure detection, seizure prediction, and closed-loop warning systems in epilepsy. Epilepsy Behav 37: 291-307. 